\title{
Modelo Didáctico en Toma de Decisiones relacionadas con la Gestión de Producción y Operaciones. Aplicación en Ingeniería Química
}

\author{
Jhonathan M. Vargas y Jaime A. Giraldo \\ Facultad de Ingeniería y Arquitectura, Universidad Nacional de Colombia Sede Manizales, \\ Carrera 27 64-60, Manizales-Colombia (e-mail: jmvargasba@unal.edu.co; jaiagiraldog@unal.edu.co)
}

Recibido Mar. 20, 2015; Aceptado May. 15, 2015; Versión final May. 27, 2015, Publicado Dic. 2015

\begin{abstract}
Resumen
Se desarrolló una herramienta de entrenamiento para estudiantes de ingeniería química con el fin de propiciar un ambiente de aprendizaje apropiado en toma de decisiones relacionadas con la gestión de producción y operaciones (GPO). Esta herramienta esta soportada en simulación de eventos discretos, mediante la cual los usuarios finales pueden plantear diferentes escenarios de operación del proceso de producción, usando como caso de estudio la producción de jabón, y evaluar como estos afectan el desempeño del sistema en términos de nivel de producción alcanzado. La herramienta desarrollada no solo aborda la producción de jabón, sino también tiene en cuenta la distribución de materias primas para el proceso y la distribución de productos terminados, entre otras decisiones abordadas por la GPO. El estudio muestra la relevancia de la simulación en los procesos de enseñanza/aprendizaje sobre todo en temas relacionados con la gestión de producción y operaciones.
\end{abstract}

Palabras clave: toma de decisiones, gestión de la producción y operaciones, ingeniería química, simulación

\section{Educational Decision Making Model related to Production and Operations Management (POM). Application in Chemical Engineering}

\begin{abstract}
A training tool for chemical engineering students with the aim of promoting a proper learning environment in decision making related to production and operations management (POM) was developed. This tool is supported on discrete event simulation, by which end users can pose different scenarios of operation of the production process, using the production of soap as a study case, and evaluates how they affect system performance in terms of production level achieved. The tool developed not only addresses the production of soap, but also takes into account the distribution of raw materials for processing and distribution of final products among other decisions addressed by the POM. The study shows the importance of simulation in the teaching / learning process, especially on issues related to the production and operations management
\end{abstract}

Keywords: decision making, production and operations management, chemical engineering, simulation 


\section{INTRODUCCIÓN}

La GPO busca la construcción y sostenibilidad de las capacidades competitivas de la unidad empresarial, lo que implica el entendimiento del papel de todas las funciones organizacionales y especialmente la producción como función clave de la misión empresarial (Cárdenas Aguirre, y otros, 2008). Al revisar el currículo de algunos programas de ingeniería industrial, ingeniería de producción e ingeniería administrativa se detectan claramente la oferta amplia de asignaturas relacionadas con la GPO, lo cual no es el caso de la ingeniería química. Más sin embargo, al menos en el contexto laboral colombiano, se le exige al ingeniero químico que se desempeña en el área de producción, un rol más activo en GPO. Evidencia de ello, lo constituye el número de estudiantes y profesionales de esta rama de la ingeniería que han cursado programas de posgrado relacionados con la GPO, el cual ha ido en aumento. Este fenómeno evidencia en un principio la necesidad que ven los ingenieros químicos por cubrir estos temas muy propios de la ingeniería industrial. Prueba de ello es que hasta el 2013 se habían registrado 33 ingenieros químicos en los posgrados referidos, en nuestra Universidad.

Según el American Institute of Chemical Engineers, (AIChE, 2014) "La ingeniería química es la profesión en la cual el conocimiento de la matemática, química y otras ciencias básicas, obtenido por el estudio, experiencia y práctica, es aplicado para desarrollar maneras económicas de usar materiales y energía para el beneficio de la humanidad". Debe connotarse en esta definición el término económico y su estrecha relación con la GPO, dado que esta última al encargarse de la planificación, organización, mejora, control y dirección de los sistemas de producción de bienes y servicios, puede garantizar que esas maneras de usar materiales y energía, sean económicas. A partir de la definicion de la profesion y la tematica abordada por la GPO, se evidencia la importancia de la GPO en ingenieria quimica y el papel de los ingenieros quimicos en la unidad empresarial. Una investigacion previa, la cual puede ser consultada en la pagina http://modelogpoeniq.wordpress.com/, evidenció que en el programa de ingenieria quimica de nuestra universidad se presenta un descuido generalizado sobre los temas relacionados con la GPO, pues se da alta prioridad al diseño (aspecto meramente tecnico) de los sistemas de produccion en detrimento de la enseñanza/aprendizaje sobre toma de decisiones operativo-gerenciales de los mismos. Ademas, dadas las dificultades de acceso para experimentar esta toma de decisiones con sistemas reales de produccion, es oportuno y relevante proponer modelos de enseñanza/aprendizaje basados en Tecnologías de la Información y las Comunicaciones (Tics) que contribuyan a procesos formativos mas integrales en ingenieria quimica.

Los procesos de producción de jabón (sistemas reales de produccion) son utilizados frecuentemente como objetos de estudio en diversos cursos en ingeniería química. Chupa, Misner, Sachdev, Wisniewski, y Smith (2012) proponen una definición general sobre el jabón como el producto formado por la saponificación o neutralización de grasas, aceites, ceras, colofonias, o sus ácidos con bases orgánicas o inorgánicas. La invención del proceso de ceniza de sosa por LeBlanc en 1791 y el descubrimiento por Chevreul en 1811 de que el jabón se compone de una mezcla de ácidos grasos, prepararon el camino a los procesos modernos de fabricación de jabón. La figura 1 muestra la cadena logística moderna en la producción de jabón. Estos sistemas en el mundo real consideran diferentes recursos, zonas de almacenamiento, equipos, diversos flujos de material y de información entre otros. Al momento de modelar estos, debe procurarse por representar sus componentes estructurales y operacionales si se quiere tener modelos válidos y representativos del sistema (Giraldo, 2014).

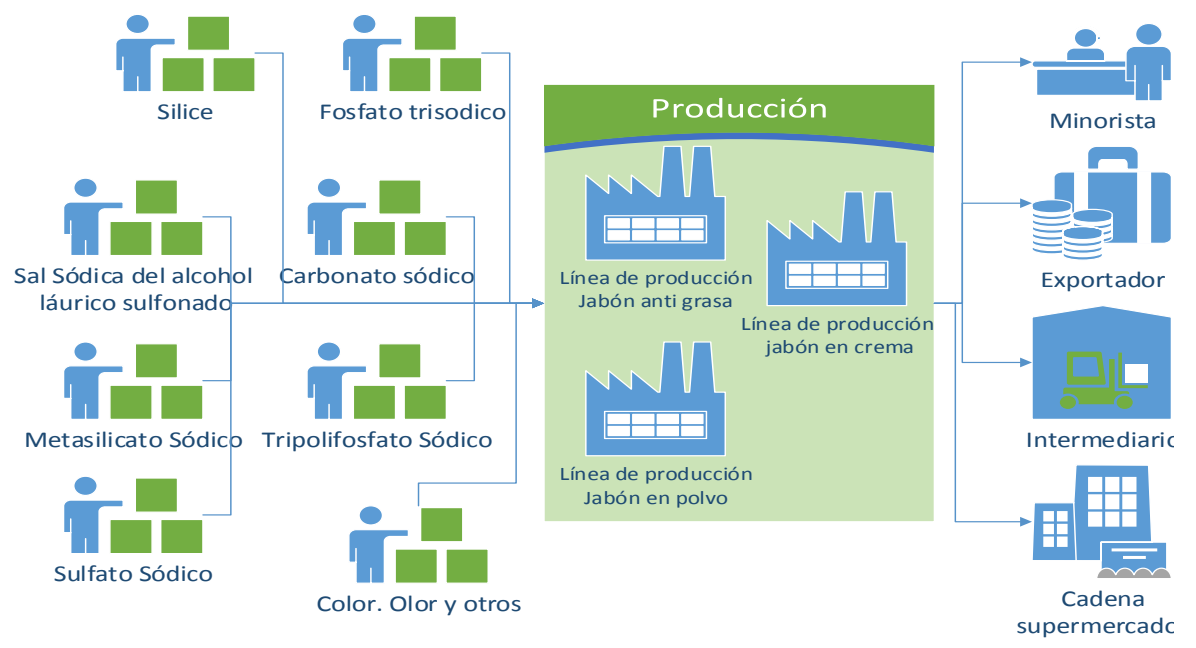

Fig. 1: Cadena logística típica en la producción de jabón. 
Desde la perspectiva de la GPO, el sistema a modelar consta estructuralmente de tres eslabones: proveedores de materia prima, planta de producción y clientes. Operacionalmente la materia prima llega a la zona de acopio, luego es trasladada a las tres líneas de producción donde se cuentan con mezcladores, empacadoras y otros equipos. El proceso es gestionado por operarios que manipulan los equipos tanto de mezclado como de empaque. El producto terminado es enviado a una zona de almacenamiento para luego ser distribuido entre los clientes. En este tipo de sistemas se deben tomar decisiones, a corto plazo como producción diaria; a mediano plazo selección de proveedores y rediseño de productos y a largo plazo requerimientos de capacidad y ubicación de la planta, entre otras. Por esta razón se propone un modelo de entrenamiento para la toma de decisiones en GPO mediante el cual se plantea una situación-problema relacionada con el desempeño operativo del sistema y cuya solución implica que el discente establezca los niveles de las variables de decisión relacionadas con la GPO (nivel de reorden de materia prima, numero de operarios en planta, capacidad de los equipos, etc.), y evaluar cómo estas impactan dicho desempeño (por ejemplo, el nivel de producción).

Al revisar la literatura sobre enfoques educativos que giren en torno a situaciones-problema, se detectan diversas publicaciones en torno al ABP (Aprendizaje Basado en Problemas). Valero-García y Navarro (2005) reportan que el modelo ABP tiene sus fundamentos en las teorías del constructivismo (Piaget, 1970) y el aprendizaje social (Vygotsky, 1986). Mesa et al. (2008) analizan la implementación de la metodología ABP en asignaturas de Dirección de Proyectos de Ingeniería. Latasa et al. (2012) concluyen que el empleo del $A B P$ en contextos curriculares no integrados produce resultados de aprendizaje superiores a los que se obtienen mediante enfoques de enseñanza-aprendizaje más tradicionales, como los centrados en la actividad del docente. De otra lado, Saez et al. (2008) utilizan ABP en el desarrollo de un solucionador de Sudoku para estudiar los conceptos relacionados con la teoría de la NP-completitud en ciencias de la computación, reportando resultados satisfactorios. Vizcarro y Juarez (2008) señalan que algunos de los factores que se deben tener en cuenta a la hora de formular un problema con enfoque ABP, son los siguientes: 1) Relevancia del problema: es un factor crítico si se quiere mantener el interés de los estudiantes además de desarrollar destrezas en ellos para transferir la habilidad y conocimiento logrados en el aula en la solución de problemas de la vida real, por lo que sugieren basar el problema en problemáticas existentes en la realidad, 2) Ámbito del problema: debe guiar a los estudiantes a descubrir/identificar la información/conocimiento requerido. Por tanto se deben identificar previamente los hechos y conceptos básicos que se espera que los estudiantes descubran al solucionar el problema, y 3) Complejidad del problema: El mundo empresarial al que se enfrentará el futuro profesional está repleto de problemas complejos, por lo que tiene sentido reflejar situaciones similares en el aula. Además, "... la complejidad ayuda a asegurar que no hay una respuesta "correcta". Teniendo múltiples respuestas correctas que abordan el problema desde diversas perspectivas y soluciones se pueden utilizar como trampolín en las discusiones de clase que estimulan al estudiante hacia un nivel superior de pensamiento".

Específicamente en el campo de la ingeniería, Valero-García y Navarro (2005), reportan un escenario de $\mathrm{ABP}$ en el cual los discentes, reciben del docente el "pliego de condiciones" del proyecto a realizar, que incluye "la definición precisa del producto a obtener y las fechas de entrega de los resultados parciales y finales". A partir de este pliego, los estudiantes identifican lo que ya saben y qué deben aprender para desarrollar el proyecto, estableciendo con la orientación del docente, un plan de aprendizaje que se ejecuta hasta que se han alcanzado los objetivos de aprendizaje, siendo lo importante el aprendizaje que se da en el proceso y no tanto el resultado final del proyecto. Estos autores hacen énfasis en que la adopción del sistema europeo de créditos (ECTS), da gran protagonismo al ABP en particular," colocando sobre la mesa del docente los dos retos siguientes: 1) Diseñar un programa de actividades para realizar dentro y fuera de clase, de las que el alumno no pueda escapar sin haber aprendido y 2) Conseguir que el alumno haga esas actividades". Es claro entonces que para lograr el primer reto, es necesario proporcionar al estudiante herramientas que le permitan realizar dichas actividades, sobre todo fuera de clase.

De otra parte, Font (2008) reporta sobre la utilización de algunas herramientas Tics que han empleado como soporte al ABP en un proyecto sobre portafolio electrónico para el aprendizaje que incluye sistemas de gestión de contenido (CMS), de trabajo en grupo (Groupware) y de entornos de enseñanza / aprendizaje (AulaWiki). Es evidente que la sociedad actual ha presentado cambios significativos en el campo de la educación y esto se debe en gran medida al uso extensivo de las Tics (Moreno, 2006). La innovación tecnológica no es ajena en los sistemas educativos, pues es objeto de constantes debates, que se dan desde afirmaciones tales como que las nuevas tecnologías han fracasado en el manejo de los estándares educativos, o por el contrario que han transformado y ampliado las universidades y sus modelos pedagógicos (Sharples y McAndrew, 2012). Aportando al debate, Santamaría y Mendoza (2014) señalan que los procesos modernos de enseñanza/aprendizaje se apoyan en recursos educativos digitales que incorporan el uso y la apropiación de las Tics. Gonzales (2014) en su blog plantea que el ABP puede soportarse en diversas herramientas TIC, las cuales clasifica en posibles actividades requeridas para lograr el aprendizaje: Organización del pensamiento (lluvia de ideas, mapas mentales/conceptuales y 
organizadores gráficos); Consumo de información ( búsqueda de información y generadores de bibliografía); Almacenamiento de información (archivos, imágenes, videos y anotaciones); Sistemas e-learning (Blackboard, Moodle); Creación/gestión de contenidos (ofimática, publicaciones, contenidos multimedia) y Evaluación del aprendizaje (mural virtual, cuestionarios y test online, diarios de aprendizaje). Consideramos que a Gonzales (2014) le falta el tener en cuenta herramientas Tics que permitan al discente experimentar virtualmente con soluciones al problema planteado. Para Fredes et al. (2012), la simulación es una herramienta que propicia un ambiente virtual y puede situar a los estudiantes en escenarios prácticos, que para nuestro caso lo constituyen la toma de decisiones en GPO. Con la simulación, los estudiantes pueden acceder a modelos validos de sistemas productivos reales y modificar las condiciones de operación. En el mismo sentido, López y Saiegg (2005) señalan que se puede utilizar la simulación de eventos discretos en los procesos de aprendizaje como una herramienta de experimentación, mejorando la forma en que los alumnos aprenden, permitiendo a los mismos trabajar con sistemas complejos y que difícilmente puedan ser estudiados de forma directa

Al enfrentarse a los retos actuales de los procesos formativos, se pueden desarrollar herramientas soportadas en Tics, en las cuales la didáctica digital y la innovación educativa cobran un papel fundamental. Giraldo et al. (2013) señalan que los procesos de enseñanza/aprendizaje son complejos y estos deben involucrar completamente a los actores que intervienen (docentes y discentes), concluyen señalando que lo ideal es que los protagonistas de estos procesos formativos no solo sean llamados a detectar problemas, sino que también hagan parte activa en el planteamiento de soluciones a los mismos. De otra parte, Morrissey (2007) señala que cada vez más el uso de las Tics aporta a las habilidades como la creatividad y la inventiva, las cuales son muy valoradas en los mercados laborales y gracias a la disponibilidad de los computadores a un menor costo, se ha llegado a una revolución social en los jóvenes. Según Van der Zee y Holkenborg (2010) en los últimos años muchos investigadores (citando a Chapman y Martin (1995), Ruohomaki (1995, 2003), Chwif y Barretto (2003), Smeds (2003), y Lainema y Hilmola (2005)) han indicado el alto potencial de los juegos con simulación para el dominio de nuevos conceptos de negocios en la gestión de operaciones.

\section{DESARROLLO METODOLÓGICO}

\section{Dificultades en el aprendizaje de la GPO en ingeniería química}

Se realizó un trabajo de campo mediante el cual se evidenciaron dificultades en la enseñanza/aprendizaje de la GPO en un programa de ingeniería química. Para este estudio se propusieron 4 categorías de variables causa-efecto que afectan dichos procesos formativos y los exploran desde diferentes puntos de vista (Vargas Barbosa y Giraldo García, 2013): i) Prácticas en los procesos de enseñanza/aprendizaje de GPO empleando TICs; ii) Contexto académico en los procesos de enseñanza/aprendizaje; iii) Características de las actividades de enseñanza/aprendizaje basadas en TICs; y iv) Presencia o ausencia de las TICs.

Para el estudio se aplicaron encuestas a estudiantes, egresados y docentes del programa de ingeniería química, como también a docentes de los posgrados relacionados con la GPO (docentes del departamento de ingeniería industrial de la misma universidad). Este estudio entre otras cosas, dejo evidenciar que los ingenieros químicos tienen falencias en el manejo de temas relacionados con la GPO, los cuales muchas veces deben ser cubiertos con posgrados relacionados. (Para ver los resultados de las encuestas, visitar el sitio http://modelogpoeniq.wordpress.com/).

\section{Conceptualización del modelo de decisión en GPO}

El planteamiento del modelo de decisión se describe mediante la expresión (1) donde la variable respuesta $y_{j}$ depende de relaciones lógico/matemáticas de las variables de decisión $x_{i, j}$ descritas por la función $f_{j}\left(x_{i, j}\right)$.

$y_{j}=f_{j}\left(x_{1, j}, x_{2, j}, x_{3, j}, x_{4, j}, x_{5, j}\right)$ donde $1 \leq j \leq 4$

Se plantean 4 variables respuesta $y_{j}$, para lo cual, cada una se ve afectada por 5 posibles (de entre muchas) variables de decisión. La descripción de variables respuesta/decisión del modelo se describen en la Tabla 1, para una instancia posible del problema con $i=5$ y $j=4$. La idea básica consiste en que el estudiante fije los niveles de las variables de decisión que el considere apropiados con el objetivo de lograr satisfacer la demanda por producto (propuesta por el docente) en un periodo de tiempo dado. A partir de simular un modelo (robusto) que representa la función $f_{j}\left(x_{i, j}\right)$, el discente puede decidir cuales serian los niveles de las variables que satisfacen la demanda de los clientes. Es claro que en la búsqueda de dichos 
niveles, el estudiante debe aprender (según la metodología ABP) sobre muy diversos temas/conceptos de la GPO, entre otros: tiempos de abastecimiento, tiempos de proceso, inventarios, demanda independiente/dependiente, programación de mano de obra y de equipos, mantenimiento preventivo/correctivo etc.

Tabla 1. Variables de decisión y sus niveles de operación.

\begin{tabular}{|c|c|c|c|c|c|}
\hline Variable respuesta & Var. & Descripción de la variable de decisión & Unidad & Máx. & Mín. \\
\hline \multirow{5}{*}{$\begin{array}{c}y_{1} \\
\text { (Producción jabón anti } \\
\text { grasa } 1 \mathrm{~kg})\end{array}$} & $x_{1,1}$ & Stock reorden Sílice jabón anti grasa $1 \mathrm{~kg}$ & $\mathrm{Kg}$ & 9000 & 18000 \\
\hline & $x_{2,1}$ & Stock reorden Empaques jabón anti grasa $1 \mathrm{~kg}$ & $\mathrm{Kg}$ & 2000 & 4000 \\
\hline & $x_{3,1}$ & Numero de montacargas dentro de la planta & Unidad & 1 & 10 \\
\hline & $x_{4,1}$ & Tiempo de mezclador sílice-fosfato trisodico & Min & 15 & 30 \\
\hline & $x_{5,1}$ & Tiempo de empaque maquina $1 \mathrm{~kg}$ & Min & 0.05 & 0.1 \\
\hline \multirow{5}{*}{$\begin{array}{c}y_{2} \\
\text { (Producción jabón anti } \\
\text { grasa } 2 \mathrm{~kg} \text { ) }\end{array}$} & $x_{1,2}$ & Stock reorden Sílice jabón anti grasa 2 kg & $\mathrm{Kg}$ & 9000 & 18000 \\
\hline & $x_{2,2}$ & Stock reorden Empaques jabón anti grasa 2 kg & $\mathrm{Kg}$ & 2000 & 4000 \\
\hline & $x_{3,2}$ & Numero de montacargas dentro de la planta & Unidad & 1 & 10 \\
\hline & $x_{4,2}$ & Tiempo de mezclador sílice-fosfato trisodico & Min & 15 & 30 \\
\hline & $x_{5,2}$ & Tiempo de empaque maquina $2 \mathrm{~kg}$ & Min & 0.05 & 0.1 \\
\hline \multirow{5}{*}{$\begin{array}{c}y_{3} \\
\text { (Producción jabón en } \\
\text { crema) }\end{array}$} & $x_{1,3}$ & Stock reorden Sílice jabón en crema & $\mathrm{Kg}$ & 9000 & 18000 \\
\hline & $x_{2,3}$ & Stock reorden Color y Olor jabón en crema & $\mathrm{Kg}$ & 200 & 400 \\
\hline & $x_{3,3}$ & Stock reorden Empaques jabón en crema & $\mathrm{Kg}$ & 2000 & 4000 \\
\hline & $x_{4,3}$ & Numero de montacargas dentro de la planta & Unidad & 1 & 10 \\
\hline & $x_{5,3}$ & Tiempo Empaque jabón en crema & Min & 0.005 & 0.01 \\
\hline \multirow{5}{*}{$\begin{array}{c}y_{4} \\
\text { (Producción jabón en } \\
\text { polvo) }\end{array}$} & $x_{1,4}$ & Stock reorden Tripolifosfato Sódico jabón en polvo & $\mathrm{Kg}$ & 700 & 1400 \\
\hline & $x_{2,4}$ & Stock reorden Empaques jabón en polvo & $\mathrm{Kg}$ & 2000 & 4000 \\
\hline & $x_{3,4}$ & Numero de montacargas dentro de la planta & Unidad & 1 & 10 \\
\hline & $x_{4,4}$ & Tiempo de mezclado olor y color jabón en polvo & Min & 20 & 40 \\
\hline & $x_{5,4}$ & Tiempo de empaque jabón en polvo & Min & 0.05 & 0.1 \\
\hline
\end{tabular}

\section{RESULTADOS Y DISCUSION}

\section{El modelo de simulación}

La representación en computadora del modelo de decisión se hizo mediante software de simulación que facilita el desarrollo de modelos robustos cercanos a la realidad estudiada, cuestión que no pasa con otras herramientas de soporte a la toma de decisiones tales como la programación lineal o matemática o algunos métodos de solución analítica como el cálculo (diferencial e integral) y el álgebra entre otros (Kelton y Law, 2007). Dicha robustez, para el caso de un sistema de produccion, implica que el modelo represente no solamente los componentes estructurales (instalaciones,maquinas, materiales, operarios), sino componentes operativos (arribos, flujos de material e informacion, tiempos de proceso, transporte, fallos), ademas de permitir estudiar el comportamiento en el tiempo del sistema modelado. En la literatura se ofrecen diversas metodologías aceptadas para la construcción de modelos y estudios con simulación. En su libro Giraldo (2014), propone una adaptación a las metodologías propuestas por Banks et al. (2010, Kelton y Law (2014) y Harrell et al. (2012), entre otros. Esta adaptación metodológica se empleó en el desarrollo del modelo de simulación.

Una etapa muy importante de la metodología es desarrollar un modelo conceptual que represente lo más aproximadamente posible el sistema que se quiere simular. La figura 2 muestra el modelo conceptual del sistema de producción del producto jabón en polvo (ver demás modelos conceptuales en http://modelogpoeniq.wordpress.com). Se ha procurado emplear en la construcción de los respectivos modelos conceptuales una simbología que permita representar tanto los componentes estructurales como operativos (robustez) del sistema de producción, además de las medidas de desempeño del sistema que se quiere estudiar. Así por ejemplo, se connota en la figura 2 símbolos que representan componentes estructurales fijos (empaque, molienda, etc.), componentes operativos (tiempos de proceso, transporte, cantidades de materia prima, etc.), además la medida de desempeño throughput que se quiere obtener en el tiempo (nivel de producción). 


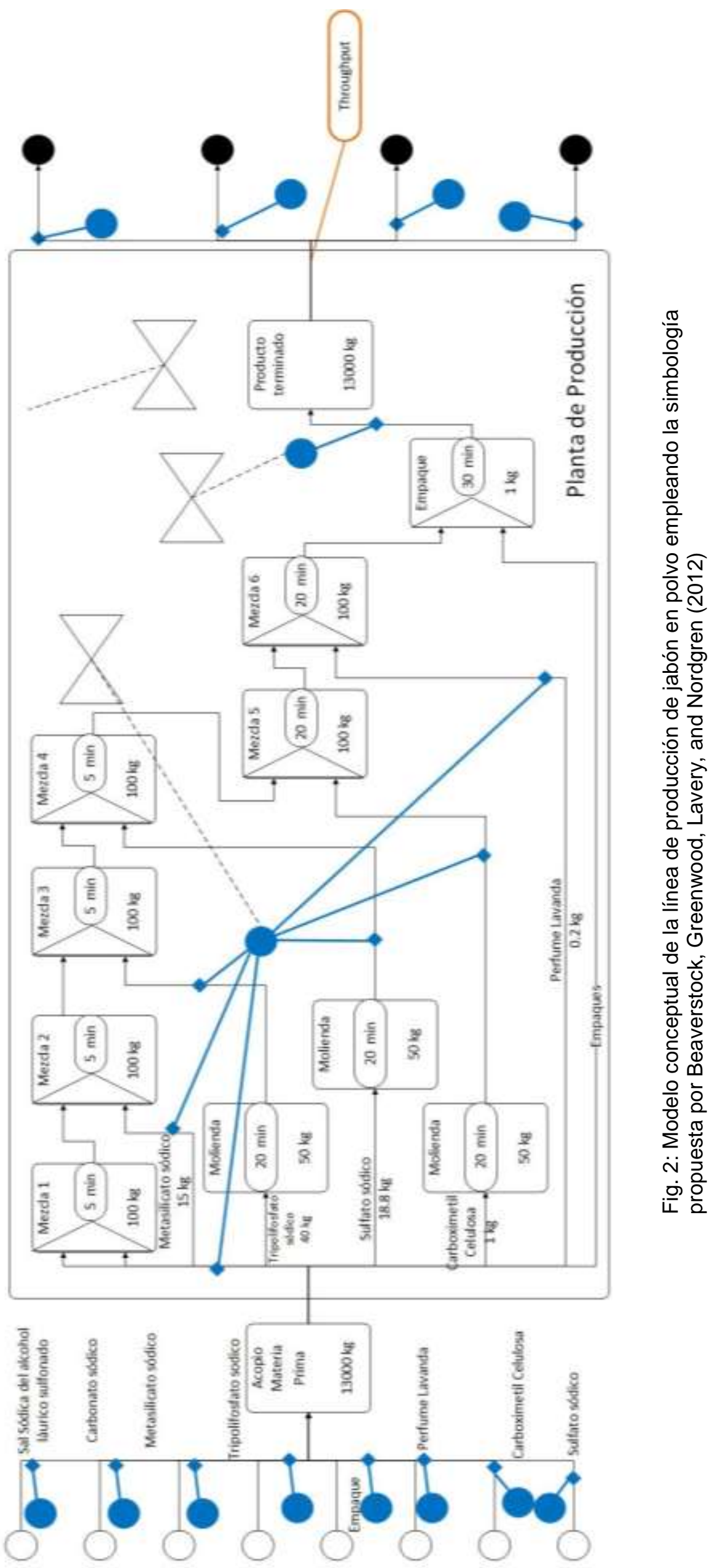


Una siguiente etapa la constituye la codificación del modelo conceptual mediante un software de simulación. Para el caso se eligió el software Flexsim en razón a su alto grado de madurez en la modelación de sistemas de producción y de servicios, además de su rápida evolución y aceptación académica. Entre las múltiples características del modelo desarrollado, se destaca que es un modelo fluido-discreto, donde no solo se modelan ocurrencias discretas, sino también una línea de producción que incluye fluidos. En la figura 3 se muestra una representación gráfica del modelo de simulación desarrollado (ver modelo completo, incluyendo el código de software y su validación en http://modelogpoeniq.wordpress.com).

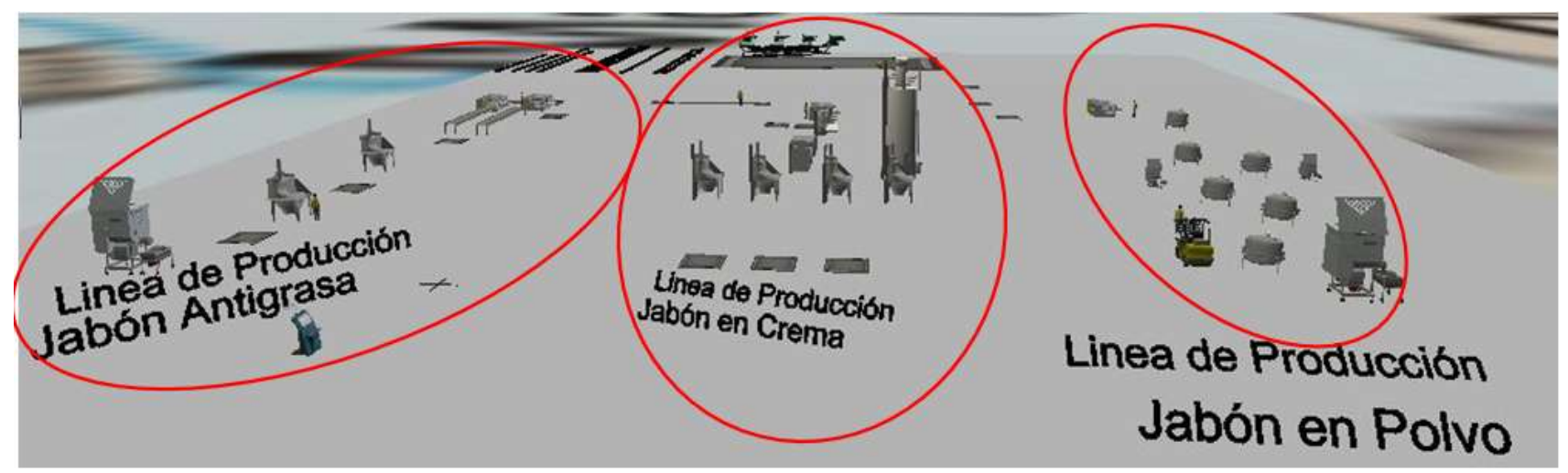

Fig. 3: Representación gráfica del modelo de simulación.

\section{Simulando instancias del problema}

A fin de lograr una interacción simple entre el discente y el modelo, se parte de que el docente proponga objetivos de producción de jabón a alcanzar en determinado tiempo (situación problema según ABP), el estudiante evalúa los diferentes escenarios (conjunto de valores dado de las variables de decisión) mediante simulación del modelo y selecciona aquel(los) que satisfacen el objetivo propuesto. La interface mostrada en la Figura 4 le permite al estudiante representar los modelos de decisión descritos en la Tabla 2 (ver las variables de decisión $x_{i, j}$ y la demanda de productos a satisfacer), además de muchos otros que el docente considere pertinentes.

Con propósitos de experimentación, se utiliza una longitud de corrida de 168 horas (1 semana) a 10 réplicas, pudiendo el usuario final modificar esta longitud de corrida según sus propios criterios. Se realizan 5 réplicas por escenario planteado para una simulación piloto, esto con el fin de conocer la variabilidad de los resultados del modelo y proponer un número de réplicas según el nivel de confianza adoptado (Giraldo García, 2014).

A fin de mostrar las potencialidades del modelo de decision, se simulo 32 escenarios a 5 replicas para las dos primeras variables respuesta (cuya situacion problema es satisfacer una demanda de 1800 y 700 unidades de producto respectivamente según se muestra en la figura 4) y sus respectivas variables de decision según la tabla 1. En la tabla 2 se presenta el comportamiento simulado de las variables respuesta produccion promedio $Y_{1}, Y_{2}$ por cada uno de los 32 escenarios. Se espera que el discente realice un analisis de la variacion del nivel de produccion según escenarios de operación (variables de decision) del sistema simulado, ademas analizar valores puntuales de produccion alcanzada. Asi por ejemplo, puntualmente en el escenario 12, se da el maximo nivel de produccion (1821) de jabon presentacion $1 \mathrm{~kg}$, esperandose que el estudiante analice los valores de decision que llevan a tan buen desempeño del sistema simulado. Para leer en detalle la configuracion de cada uno de los escenarios y los resultados obtenidos por cada replica, el lector puede visitar la pagina http://modelogpoeniq.wordpress.com/.

\section{CONCLUSIONES}

De los resultados y el análisis expuesto se pueden obtener las siguientes conclusiones sobre el Modelo Didáctico en Toma de Decisiones en GPO:

El modelo desarrollado garantiza que se han tenido en cuenta simultáneamente las siguientes concepciones en su diseño: Educacional, Comunicacional y Computacional. Desde el punto de vista educacional se ha considerado el $\mathrm{ABP}$, computacionalmente emplea la simulación discreta y la comunicación usuario-modelo se hace a través de una interface que permite manipular diversas variables de respuesta/decisión. 


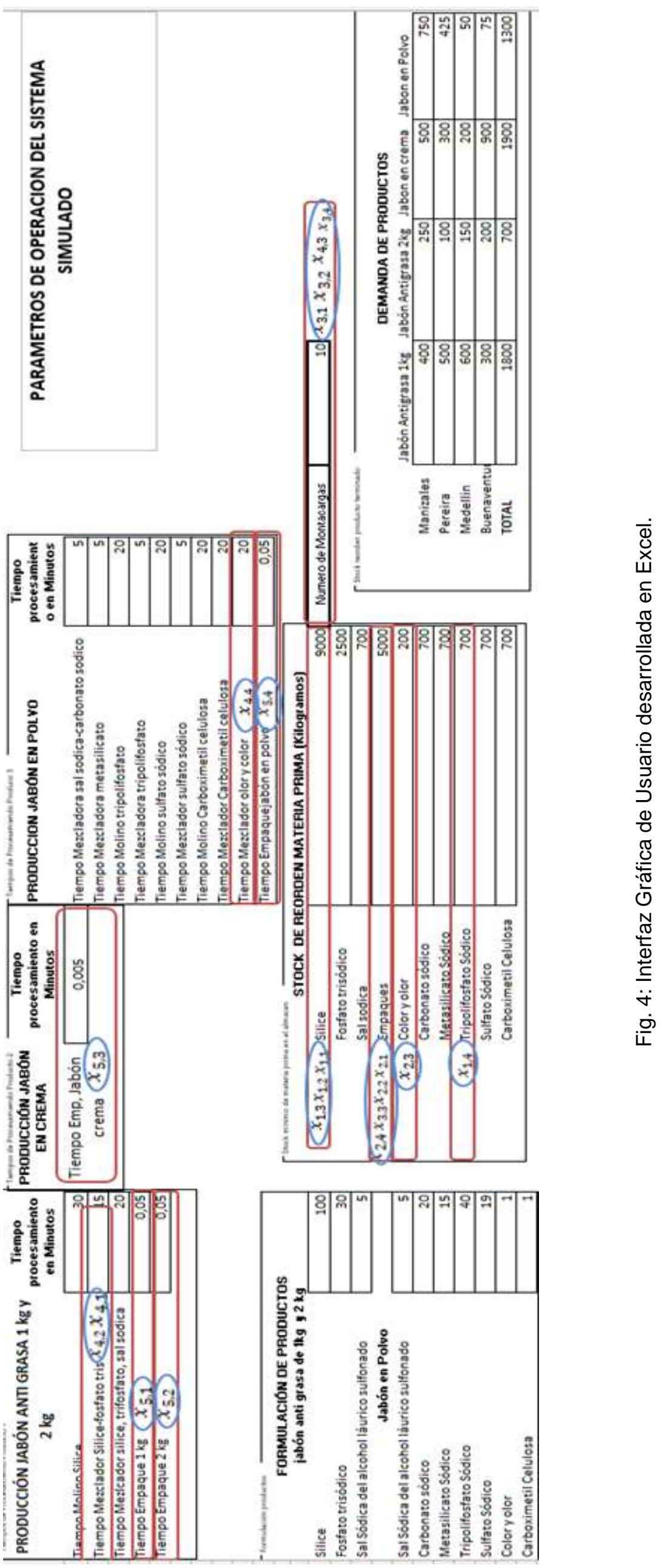


Tabla 2. Variación de la producción de jabón para cada escenario planteado

\begin{tabular}{|c|c|c|c|c|c|c|}
\hline \multicolumn{7}{|c|}{$y_{1}$ (producción jabón anti grasa 1kg) } \\
\hline Escenarios & $x_{1,1}$ & $x_{2,1}$ & $x_{3,1}$ & $x_{4,1}$ & $x_{5,1}$ & $\begin{array}{l}\text { Prod. } \\
\text { Prom. }\end{array}$ \\
\hline 1 & 9000 & 2000 & 1 & 15 & 0,05 & 1335,6 \\
\hline 2 & 18000 & 2000 & 1 & 15 & 0,05 & 1335,6 \\
\hline 3 & 9000 & 4000 & 1 & 15 & 0,05 & 1282,4 \\
\hline 4 & 18000 & 4000 & 1 & 15 & 0,05 & 1307,2 \\
\hline 5 & 9000 & 2000 & 10 & 15 & 0,05 & 633,8 \\
\hline 6 & 18000 & 2000 & 10 & 15 & 0,05 & 571,6 \\
\hline 7 & 9000 & 4000 & 10 & 15 & 0,05 & 935,2 \\
\hline 8 & 18000 & 4000 & 10 & 15 & 0,05 & 937 \\
\hline 9 & 9000 & 2000 & 1 & 30 & 0,05 & 1139,8 \\
\hline 10 & 18000 & 2000 & 1 & 30 & 0,05 & 1487,2 \\
\hline 11 & 9000 & 4000 & 1 & 30 & 0,05 & 1372,6 \\
\hline 12 & 18000 & 4000 & 1 & 30 & 0,05 & 1821 \\
\hline 13 & 9000 & 2000 & 10 & 30 & 0,05 & 894,2 \\
\hline 14 & 18000 & 2000 & 10 & 30 & 0,05 & 592,8 \\
\hline 15 & 9000 & 4000 & 10 & 30 & 0,05 & 1075,2 \\
\hline 16 & 18000 & 4000 & 10 & 30 & 0,05 & 993,2 \\
\hline 17 & 9000 & 2000 & 1 & 15 & 0,1 & 1547,4 \\
\hline 18 & 18000 & 2000 & 1 & 15 & 0,1 & 1455,6 \\
\hline 19 & 9000 & 4000 & 1 & 15 & 0,1 & 1594,2 \\
\hline 20 & 18000 & 4000 & 1 & 15 & 0,1 & 1594,2 \\
\hline 21 & 9000 & 2000 & 10 & 15 & 0,1 & 1080 \\
\hline 22 & 18000 & 2000 & 10 & 15 & 0,1 & 1090 \\
\hline 23 & 9000 & 4000 & 10 & 15 & 0,1 & 1017,8 \\
\hline 24 & 18000 & 4000 & 10 & 15 & 0,1 & 1269,2 \\
\hline 25 & 9000 & 2000 & 1 & 30 & 0,1 & 1293,6 \\
\hline 26 & 18000 & 2000 & 1 & 30 & 0,1 & 654,8 \\
\hline 27 & 9000 & 4000 & 1 & 30 & 0,1 & 882,2 \\
\hline 28 & 18000 & 4000 & 1 & 30 & 0,1 & 1423,2 \\
\hline 29 & 9000 & 2000 & 10 & 30 & 0,1 & 1083,2 \\
\hline 30 & 18000 & 2000 & 10 & 30 & 0,1 & 898,6 \\
\hline 31 & 9000 & 4000 & 10 & 30 & 0,1 & 976 \\
\hline 32 & 18000 & 4000 & 10 & 30 & 0,1 & 1135 \\
\hline
\end{tabular}

\begin{tabular}{|c|r|r|r|r|r|r|}
\hline \multicolumn{7}{|c|}{$y_{2}$ (producción jabón anti grasa $2 \mathrm{~kg}$ ) } \\
\hline Escenarios & $x_{1,2}$ & $x_{2,2}$ & $x_{3,2}$ & $x_{4,2}$ & $x_{5,2}$ & $\begin{array}{r}\text { prod. } \\
\text { prom. }\end{array}$ \\
\hline 1 & 9000 & 2000 & 1 & 15 & 0,05 & 566,2 \\
\hline 2 & 18000 & 2000 & 1 & 15 & 0,05 & 566,2 \\
\hline 3 & 9000 & 4000 & 1 & 15 & 0,05 & 497,2 \\
\hline 4 & 18000 & 4000 & 1 & 15 & 0,05 & 497 \\
\hline 5 & 9000 & 2000 & 10 & 15 & 0,05 & 355 \\
\hline 6 & 18000 & 2000 & 10 & 15 & 0,05 & 365,6 \\
\hline 7 & 9000 & 4000 & 10 & 15 & 0,05 & 424,6 \\
\hline 8 & 18000 & 4000 & 10 & 15 & 0,05 & 423,6 \\
\hline 9 & 9000 & 2000 & 1 & 30 & 0,05 & 589,6 \\
\hline 10 & 18000 & 2000 & 1 & 30 & 0,05 & 696,8 \\
\hline 11 & 9000 & 4000 & 1 & 30 & 0,05 & 617,2 \\
\hline 12 & 18000 & 4000 & 1 & 30 & 0,05 & 762,2 \\
\hline 13 & 9000 & 2000 & 10 & 30 & 0,05 & 459 \\
\hline 14 & 18000 & 2000 & 10 & 30 & 0,05 & 372,2 \\
\hline 15 & 9000 & 4000 & 10 & 30 & 0,05 & 502,8 \\
\hline 16 & 18000 & 4000 & 10 & 30 & 0,05 & 475 \\
\hline 17 & 9000 & 2000 & 1 & 15 & 0,1 & 640,4 \\
\hline 18 & 18000 & 2000 & 1 & 15 & 0,1 & 621,6 \\
\hline 19 & 9000 & 4000 & 1 & 15 & 0,1 & 627,6 \\
\hline 20 & 18000 & 4000 & 1 & 15 & 0,1 & 627,6 \\
\hline 21 & 9000 & 2000 & 10 & 15 & 0,1 & 559,4 \\
\hline 22 & 18000 & 2000 & 10 & 15 & 0,1 & 549,4 \\
\hline 23 & 9000 & 4000 & 10 & 15 & 0,1 & 574,4 \\
\hline 24 & 18000 & 4000 & 10 & 15 & 0,1 & 616,6 \\
\hline 25 & 9000 & 2000 & 1 & 30 & 0,1 & 621,6 \\
\hline 26 & 18000 & 2000 & 1 & 30 & 0,1 & 405,8 \\
\hline 27 & 9000 & 4000 & 1 & 30 & 0,1 & 520,2 \\
\hline 28 & 18000 & 4000 & 1 & 30 & 0,1 & 700,4 \\
\hline 29 & 9000 & 2000 & 10 & 30 & 0,1 & 520,6 \\
\hline 30 & 18000 & 2000 & 10 & 30 & 0,1 & 471,2 \\
\hline 31 & 9000 & 4000 & 10 & 30 & 0,1 & 598 \\
\hline 32 & 18000 & 4000 & 10 & 30 & 0,1 & 666,2 \\
\hline & & & & & \\
\hline & & & &
\end{tabular}

Al considerar el Aprendizaje Basado en Problemas (diseño educacional), se espera que al usar el modelo propuesto: 1) se motive e implique a los estudiantes en el aprendizaje de la GPO; 2) conlleve a estos a reflexionar sobre el proceso de aprendizaje de la GPO y 3) logre que los discentes profundicen en los conocimientos alrededor de la GPO y desarrollen la capacidad de aplicar estos conocimientos en su ejercicio profesional, cuando resulten pertinentes. Entre las cualidades más importantes del modelo de simulación desarrollado (diseño computacional) se destaca la representación completa de la cadena de producción de jabón, desde el abastecimiento, pasando por la producción e incluyendo la distribución. Igualmente es de connotar la representación simultánea de procesos con unidades discretas y fluidas, los cuales son de interés en ingeniería quimica. Finalmente, la simplicidad del diseño comunicacional del modelo (ver figura 4) permite una fácil experimentación, sobre todo para diseños factoriales $2^{k}$ e incluso $3^{k}$.

\section{AGRADECIMIENTOS}

Los autores agradecen a la Universidad Nacional de Colombia sede Manizales a través de la dirección de investigaciones (DIMA) por su apoyo al desarrollo de esta investigación (proyecto: "Modelo de soporte al proceso de enseñanza/aprendizaje de la Gestión de la Producción y Operaciones basado en TICs. Aplicación al programa de Ingeniería Química Etapa 2" código Hermes 28278).

\section{REFERENCIAS}

American Institute of Chemical Engineers (AIChE), AIChE Constitution, http://www.aiche.org/ about/governance/constitution, Acceso: 8 Junio (2014) 
Beaverstock, Greenwood, Lavery, y Nordgren, Applied Simulation Flexsim with Software, Flexsim Software Products, Inc, (2012)

Cárdenas Aguirre, D. M., Castrillón Gómez, Ó. D., Becerra Rodríguez, F., Giraldo García, J. A., Ibarra Mirón, S., y Zapata Gómez, A., Gestión De La Producción: Una Aproximación Conceptual. Bogotá: Universidad Nacional de Colombia, Unibiblos (2008)

Chupa, J., Misner, S., Sachdev, A., Wisniewski, P., y Smith, G. A., 36. Soap, Fatty Acids, and Synthetic Detergents. En J. A. Kent, Handbook of Industrial Chemistry and Biotechnology. 1431-1471. New York: Springer Science+Business Media, (2012)

FlexSim Software Products, Inc., What is FlexSim simulation software?, https://www.flexsim.com/, Acceso: 18 de Noviembre (2014)

Fredes, C. A., Hernández, J. P., y Diaz, D. A., Potencial y Problemas de la Simulación en Ambientes Virtuales para el Aprendizaje, doi: 10.4067/S0718-50062012000100006, Form. Univ.,5, 45-56 (2012)

Giraldo García J. A., Simulación de Sistemas de Producción y de Servicios, Universidad Nacional de Colombia, Facultad de Ingeniería y Arquitectura, Manizales (2014)

Giraldo, J. A., Toro, C. A., y Jaramillo, F. A., Aprendiendo sobre la Secuenciación de Trabajos en un Job Shop Mediante el uso de Simulación, doi: 10.4067/S0718-50062013000400004 Form. Univ., 6, 27-38 (2013)

Gonzalez, J. Principia Enseñando y Aprendiendo con Tecnología, http://blog.princippia.com/2014/07/ aprendizaje-basado-problemas-con-las-tic.html?m=1, Acceso: 17 Febrero (2014)

Herrera Gallego, I., Gestión Moderna de Producción Aplicando la Teoría de Restricciones. Manizales: Artes Gráficas Tizan, (2003)

Kelton, W. D., y Law, A., Simulation Modeling and Analysis 3nd Ed. McGraw-Hill, (2007)

L. Santamaría, y Mendoza, J. F., Construcción de Mundos Virtuales para el Desarrollo de Destrezas de Lateralidad Basado en Web3D; ISSN 1900-8260. Revista Educación en Ingeniería, 13-25 (2014)

Latasa, Itxaro, Lozano, Peio, y Ocerinjauregi, Nagore., Aprendizaje Basado en Problemas en Currículos Tradicionales: Beneficios e Inconvenientes, doi: 10.4067/S0718-50062012000500003 Formación universitaria, 5(5), 15-26. (2012)

López, C. A., y Saiegg, C. A., Uso de la Simulación como Estrategia de Mejora en el Proceso de Enseñanza-Aprendizaje en las Universidades. Una Aplicación para la Carrera de Informática. Tesis Licenciatura en Informática. Comodoro Rivadavia: Univ. Nacional de la Patagonia San Juan Bosco, (2005)

Mesa, José M, Álvarez, José V, Villanueva, Joaquín M, y de Cos, Francisco J., Actualización de Métodos de Enseñanza-Aprendizaje en Asignaturas de Dirección de Proyectos de Ingeniería. doi: 10.4067/S071850062008000400004, Form. Univ., 1(4), 23-28 (2008)

Moreno, M. E., Las TIC y el Desarrollo del Aprendizaje en Educación Inicial. http://goo.gl/HbKHga Revista Electronica de Humanidades, Educación y Comunicación Social, 1-11 (2006)

Morrissey, J., El uso de TIC en la enseñanza y el aprendizaje. Cuestiones y desafíos. En Unicef, Las TIC: del aula a la agenda política, 81-90, Buenos Aires (2007)

Proexport Colombia, Sector Cosméticos y Artículos de Aseo. Colombia. Bogotá, (2012)

Sáez, Pablo D, y Monsalve, César E., Aprendizaje Basado en Resolución de Problemas en Ingeniería Informática, doi: 10.4067/S0718-50062008000200002, Formación universitaria, 1(2), 3-8. (2008)

Sharples, M., y McAndrew, P., Innovating Pedagogy. Exploring New Forms of Teaching, Learning and Assessment, too Guide Educators and Policy Makers. United Kingdom: The Open University, (2012)

Valero-García M., y Navarro J., La planificación del trabajo del estudiante y el desarrollo de su autonomía en el aprendizaje basado en proyectos, en La Metodología del Aprendizaje Basado en Problemas, Univ. Politécnica de Cataluña (2005)

Van der Zee, D. J., Conceptual Modelling for Simulation-Based Serious Gaming. Procedente de Winter Simulation Conference, 522-534. USA 5 al 8 de diciembre (2010)

Vargas Barbosa, J. M., Proyecto de Tesis de Maestría: Modelo de Soporte al Proceso Enseñanza/Aprendizaje de la Gestión de la Producción y Operaciones basado en TIC. Aplicación al Programa de Ingeniería Química. Manizales: Universidad Nacional de Colombia sede Manizales, . (2013)

Vizcarro C. y Juárez E.., ¿Qué es y cómo funciona el aprendizaje basado en problemas?, en La Metodología del Aprendizaje Basado en Problemas. Murcia: Editum, Ediciones de la Universidad de Murcia (2008) 